\title{
GERENCIANDO INTANGIBLES EN EMPRESAS DE SOFTWARE APLICANDO EL PROCESO DE ANÁLISIS POR JERARQUÍAS Y EL CUADRO DE MANDO INTEGRAL*
}

\author{
PATRICIA GONZÁLEZ GONZÁLEZ** \& TATIANA BERMÚDEZ RODRÍGUEZ*** \\ UNIVERSIDAD DEL VALLE
}

Recibido/ Received/ Recebido: 24-08-2010 - Aceptado/ Accepted / Aprovado: 23-05-2011

\begin{abstract}
Resumen
El objetivo de este artículo es presentar una metodología que a partir de la integración de dos herramientas como son el Analytic Hierarchy Process (AHP) y el Balanced Scorecard (BSC), permita gerenciar los intangibles en empresas basadas en el conocimiento como es el caso de las empresas de software. Se efectuó un estudio de caso, cuyos resultados permitieron identificar los intangibles que son prioritarios para este tipo de empresas. Por otro lado, se observó coherencia entre los intangibles prioritarios y la especificidad que cada una de las empresas presenta en cuanto a su razón de ser: diseño, mantenimiento y comercialización. Se concluye que la integración del AHP y el BSC da origen a una metodología que facilita la gestión de los intangibles, a partir de la jerarquización y monitoreo de desempeño y logro de los mismos expresados en objetivos estratégicos e indicadores en empresas de software.
\end{abstract}

Palabras clave: Gestión, Intangibles, Proceso de análisis por jerarquías, Cuadro de mando integral, Empresas de Software.

\section{MANAGING INTANGIBLES IN SOFTWARE COMPANIES APPLYING ANALYTIC HIERARCHY PROCESS AND BALANCED SCORECARD}

\begin{abstract}
The objective of this article is to present a methodology that uses two tools such as Analytic Hierarchy Process (AHP) and Balanced Scorecard (BSC), and also it allows managing intangibles in a company based on knowledge as software companies. A study case was made, which results allowed identifying intangibles necessary for this kind of companies. On the other hand, there was coherence among necessary intangibles and the specificity of each company according its purpose: design, maintenance and commercialization. This concludes that AHP and BSC integration origins a methodology that eases intangible management from hierarchy and performance monitoring and achievement expressed on strategic objectives and indicators in software companies.

Keywords: Management, intangibles, Analytic Hierarchy Process (AHP), Balanced Scorecard, Software companies.
\end{abstract}

* Artículo resultado del proyecto de investigación: "Propuesta de un abordaje para la valoración y evidencia del activo intangible, en empresas pymes de innovación tecnológica que desarrollan software en Cali, Colombia". Universidad del Valle.

* Doctora en Contabilidad, profesora Titular y Directora del Grupo de Investigación Temas Contemporáneos en Contabilidad, Control, Gestión y Finanzas. Categoría A1 de Colciencias. Facultad de Ciencias Sociales y Económicas, Universidad del Valle.

Correo electrónico: patricia.gonzalez@correounivalle.edu.co

** Magíster en Ingeniería Industrial y Economista Universidad del Valle, Asistente de Investigación Grupo de Temas Contemporáneos en Contabilidad, Control, Gestión y Finanzas, Facultad de Ciencias Sociales y Económicas, Universidad del Valle. Correo electrónico: tatyber79@hotmail.com 


\title{
A GERÊNCIA DE BENS INTANGÍVEIS EM EMPRESAS DE SOFTWARE APLICANDO O PROCESSO DE ANÁLISE POR HIERARQUIAS E O QUADRO DE COMANDO INTEGRAL
}

\begin{abstract}
Resumo
O objetivo deste artigo é apresentar uma metodologia que a partir da integração de duas ferramentas como Analytic Hierarchy Process (AHP) e Balanced Scorecard (BSC), permita gerenciar os bens intangíveis em empresas baseadas no conhecimento como é o caso das empresas de software. Efetuou-se um estudo de caso, cujos resultados permitiram identificar os bens intangíveis que são prioritários para este tipo de empresas. Por outro lado, observou-se coerência entre os bens intangíveis prioritários e a especificidade que cada uma das empresas apresenta quanto a sua razão de ser: desenho, manutenção e comercialização. Conclui-se que a integração do AHP e o BSC dá origem a uma metodologia que facilita a gestão dos bens intangíveis a partir da hierarquização e monitoramento de desempenho e lucro dos mesmos, expressados em objetivos estratégicos e indicadores em empresas de software.
\end{abstract}

Palavras chave: Gestão, Intangíveis, Analytic Hierarchy Process (AHP), Balanced Scorecard, Empresas de Software.

González, P. \& Bermúdez, T. (2011) Gerenciando intangibles en empresas de software aplicando el proceso de análisis por jerarquías y el cuadro de mando integral. En: Revista de la Facultad de Ciencias Económicas de la Universidad Militar Nueva Granada. rev.fac.cienc.econ, XIX (2)

JEL: M41, M49.

\section{Introducción}

A pesar de que se han realizado muchos esfuerzos, por parte de investigadores y empresas, para definir a los intangibles e identificar una metodología apropiada para su medición, se puede decir que aún no existe un consenso al respecto.

Los usuarios encuentran grandes dificultades en el momento de darle un valor o de medir los intangibles, una vez que existe un desconocimiento sobre los potenciales medios que faciliten dicho proceso. No obstante existe la herramienta de la matemática conocida como el Proceso de análisis por jerarquías "Analytic Hierarchy Process" (AHP), que puede contribuir con el proceso de la gestión de intangibles al interior de organizaciones basadas en el conocimiento, como es el caso de las empresas de software.

El AHP forma parte del proceso de gestión de un intangible, a partir de la priorización o jerarquiza- ción de los mismos y por medio del cálculo de un vector de prioridad, el cual es calculado a partir de la percepción de un equipo multidisciplinario que participa en el cálculo de dicho vector. Por su parte, el Balanced Scorecard (BSC), permite traducir los intangibles categorizados por el AHP, en objetivos estratégicos, que luego se evidencian en un mapa de valor dentro de las cuatro perspectivas que lo conforman y que para fines de gestión se trasladan a un tablero de mando.

En consideración a lo expuesto, en este artículo se trata en su primera parte, la importancia de la identificación y jerarquización de los intangibles; en la segunda parte, se presenta el marco conceptual en el cual se definen intangibles, AHP y BSC; en la tercera parte se explica la metodología empleada; en la cuarta parte se expone el desarrollo de un caso de estudio y los resultados obtenidos y finalmente se presentan algunas consideraciones. 


\section{Importancia de la identificación y jerarquización de los intangibles empresariales}

En los últimos años, los intangibles se han considerado como los activos más importantes para muchas empresas en el mundo, ya que se han convertido en un medio que permite a las empresas ganar ventajas competitivas garantizando con ello la rentabilidad y continuidad de sus operaciones en el corto y mediano plazo. A pesar de todo ello, las empresas y los usuarios, en general, desconocen la forma de cómo medirlos o valorarlos. Una segunda razón radica en que la medición de los intangibles tiene un impacto en el comportamiento de los individuos, especialmente, en los casos en que los resultados de la medición se vinculan con el logro de metas y compensaciones. En estas circunstancias, los resultados de la medición son considerados como indicadores $e$ inductores de desempeño de largo plazo. Una tercera razón, identificada por Marr et al., (2003) se relaciona con la evaluación de indicadores de desempeño y de ventaja competitiva que mejoran la toma de decisiones estratégicas.

Por otro lado, las normas de contabilidad aplicadas para valorar tales intangibles, generalmente, no ayudan a medirlos y menos a valorarlos de una manera razonable, de tal forma que estos en muchas ocasiones son subvalorados, sub-gerenciados o sub explotados. (King, 2003). A pesar de de la importancia y complejidad de los intangibles, al interior de las organizaciones existe una poca coordinación entre los profesionales que están encargados de ellos. Por lo tanto King $(2003,1)$ sugiere que para entender mejor los intangibles al interior de una compañía, deberían ser contestadas las siguientes preguntas:

- “¿Cuáles son los intangibles usados en el negocio?

- ¿Cuál es su valor? (según el nivel de riesgo).

- ¿Quién es el propietario?

- ¿Cómo podría ser la mejor manera para explotarlo? (por ejemplo, en la concesión de licencias o en el caso de licencias de tecnología).

- ¿Cuál el nivel de riesgo para el activo intangible?".
Uno de los factores que afectan el éxito o el fracaso de un negocio es aquel que se relaciona con la explotación del activo intangible y el cálculo del valor del riesgo. Por lo tanto, la gerencia necesita medir y conocer el valor de todos los activos y pasivos que aparecen en los estados financieros y que están bajo su control. Por otro lado, la explotación de un activo intangible puede tomar muchas formas que van desde la venta de un activo, joint venture o un acuerdo de licencia, inevitablemente, la explotación aumenta el riesgo valorado del activo. La valoración del activo permite relacionar dos conceptos importantes como son el económico y el legal del concepto de propiedad. La presencia de un activo es una función de su capacidad para generar un retorno y la tasa de descuento aplicada al retorno.

King $(2003,1)$ considera que la regla cardinal de la valoración comercial es: "el valor de alguna cosa, el cual no puede ser fijado en el abstracto, esto solo se podrá realizar, si esa cosa existe en algún lugar, en un tiempo y circunstancia en particular". No obstante, el autor considera que a lo anterior debería adicionarse las siguientes preguntas para la respectiva medición y valoración comercial: ¿para qué propósito? y ¿para quién?

En ese sentido, es evidente que la medición y valoración de los intangibles es de suma importancia para las empresas, ya que en la medida que sea valorado y medido adecuadamente, este impactará no solo la presentación de las cifras en los estados financieros, como la elaboración de nuevos estados complementarios para un mayor entendimiento y toma de decisiones estratégicas al interior de las organizaciones, respectivamente.

\section{Marco conceptual}

\subsection{Definición de Intangible desde diversos abordajes}

La mayoría de las definiciones coinciden en que los intangibles son fuentes generadoras de probables beneficios futuros, carentes de sustancia física y se encuentran controlados por la empresa como resul- 
tado de previos eventos o transacciones. (Belkaoui, 1992; Egginton, 1990; Arthur Andersen, 1992; Stickney \& Weil 1994; Cañibano et. al, 1999; Lev, 2003; Chiesa et. al, 2005; Marr, 2007, Cañibano, 2000).

Por otro lado, existen otras consideraciones que huyen un poco de la definición general que podemos encontrar en relación a este activo: Para Cañibano et al. (1999), los intangibles tienen su origen en los conocimientos, habilidades, valores y actitudes de las personas. Se consideran intangibles, las capacidades que se generan en la organización, cuando los recursos empiezan a trabajar en grupo. El conocimiento asociado a una persona y a una serie de habilidades personales se convierte en sabiduría y, finalmente, el conocimiento asociado a una organización y a una serie de capacidades organizativas se convierte en Capital Intelectual.

Para Hendriksen (1982), la principal diferencia entre los activos tangibles e intangibles radica en la incertidumbre que existe en cuanto a la capacidad de generar beneficios futuros. Por otro lado, para Hendriksen \& Van Breda (1992), los intangibles aparecen cuando se invierte dinero en efectivo u otros medios de pago equivalentes en la adquisición del servicio. Estos autores además de reconocer los intangibles tradicionales, también, consideran que dentro de este grupo podrían incluirse algunos tipos de pagos anticipados.

Siguiendo esa línea encontramos que la OCDE (Organización para la Cooperación y el Desarrollo Económico), en 1992 consideró que los desembolsos en intangibles comprenden todos aquellos realizados a largo plazo por una empresa con el fin de mejorar su situación futura, no destinados a la adquisición de activos fijos (OECD, 1992 a; 1992 b).

Volsselman (1998) define a los intangibles desde un enfoque operativo caracterizándolos como gastos corrientes en productos intangibles que están disponibles en el período y cuyo uso se extiende durante más de un año. Para este autor suele ser difícil diferenciar entre una inversión y un costo operativo, para el caso de los intangibles, debido a que están relacionados con servicios.
Los intangibles, incluyendo el "Goodwill", son frecuentemente considerados como la diferencia entre el precio de adquisición de una compañía y el valor neto contable de sus activos tangibles, siendo entonces valores residuales que finalmente vienen a representar la parte del precio de adquisición que no puede ser atribuida a otros activos tangibles (Cañibano, et al, 1999; White, Sondhi \& Fried, 1994).

Los intangibles de la organización, si son gestionados y medidos convenientemente, se transforman en una fuente de ventaja competitiva sostenible capaz de proporcionar valor organizativo y se traducen en beneficios importantes (Cañibano et al, 1999). Para Lev $(2004,2)$, "los intangibles (empleados calificados, patentes y Know-How, software, relaciones sólidas con los clientes, marcas, procesos y modelos organizacionales únicos, entre otros), generan la mayor parte del crecimiento empresarial y del valor para los accionistas. Representan más de la mitad de la capitalización de mercado de las empresas que cotizan en bolsa. De hecho, estos activos "blandos" son los que brindan a las empresas su ventaja competitiva concreta".

Según Dierickx \& Cool (1993) citado en Ordoñez (2000) los intangibles deben satisfacer los siguientes requisitos para ser considerados estratégicos:

- no se deben comercializar.

- se desarrollan y acumulan dentro de la empresa.

- tienen un fuerte carácter tácito y complejidad social.

- $\quad$ su origen se da a partir de las habilidades y aprendizaje organizativo.

- $\quad$ es inmóvil y está vinculado a la empresa.

- su desarrollo es "dependiente de la senda", es decir, depende de los niveles de aprendizaje, inversión, inventarios de activos y actividades de desarrollo previas.

Reilly (1994, 26) menciona algunos atributos que deben tener los intangibles para que sean susceptibles de ser valorados deben:

- "ser identificados específicamente y tener una descripción reconocible. 
- estar sujetos a una protección y a una existencia legal.

- estar sujetos a derechos de propiedad privada $y$ estos derechos deben ser legalmente transferibles.

- tener alguna evidencia tangible o una manifestación de la existencia de intangible (Por ejemplo, un contrato, una licencia o un documento registrado).

- $\quad$ estar sujetos a ser destruidos o terminar su existencia en un tiempo identificable o como resultado de un evento identificable".

En otras palabras, deben tener derechos legales (y otras propiedades naturales) asociados con la existencia de algún activo intangible.

De esta manera, la concepción de intangible tiene características muy particulares que merecen ser estudiadas a profundidad y tenidas en cuenta en el momento de hacer su medición/valoración, al interior de una organización. Uno de los problemas fundamentales en relación a los intangibles es la dificultad que existe para identificarlos por separado de otros activos o gastos, lo que llevaría a pensar que no cumplen con un requisito fundamental para su reconocimiento contable. Del mismo modo surgen inquietudes en cuanto a la manera de reconocer su desgaste, pues al no tener sustancia física, difícilmente se podrían depreciar empleando los mismos métodos que se usan para depreciar los activos tangibles (Cañibano et. al, 1999).

Otro problema relacionado con los intangibles es que la contabilidad tradicional no refleja con precisión el valor de los mismos. Los estados financieros no proporcionan información de todos los activos y pasivos de la empresa, sino que sólo refleja aquellos que cumplen con ciertas condiciones. Esto podría generar que no todos los intangibles sean evidenciados y mucho menos valorados adecuadamente cuando se presentan en los estados financieros. (SFAC 5, FASB ${ }^{1}$, Cañibano et al. 1999). Para Hendriksen \& Van Breda (1992), en consideración a los problemas detectados han sugerido que los métodos de valoración tradicionales aplicados a los activos tangibles podrían no ser los más apropiados para los intangibles.

Lev (2004) plantea que las empresas y los organismos contables deberían realizar un esfuerzo sistemático por desarrollar nuevos modelos de información y valoración. Estos deben ser capaces de reflejar confiablemente las características distintivas de los intangibles. Estos atributos incluyen:

- Los enormes beneficios potenciales que los intangibles pueden generar, que suelen materializarse solo tras largos periodos de inversión y desarrollo.

- La incertidumbre que rodea al resultado de invertir en intangibles.

- La exposición de los competidores a infracciones, debido al limitado alcance de la protección de derechos de propiedad que entregan los intangibles.

- La inexistencia de mercados activos que guíen la valoración de intangibles y que proveen a los inversionistas de estrategias de salida.

\subsection{El proceso de análisis de jerarquías Analytic Hierarchy Process (AHP)}

La herramienta Analytic Hierarchy Process (AHP) o Proceso de análisis de jerarquías, fue desarrollada por el Thomas L. Saaty a finales de la década de los 70. Esta herramienta permite la descomposición de un problema en niveles de jerarquías asegurando que los aspectos cuantitativos como cualitativos de dicho problema sean incorporados en el proceso de evaluación, durante el cual la opinión es extraída sistemáticamente por medio de comparaciones entre pares.

El AHP permite la aplicación de datos, experiencias, conocimiento e intuición de una forma lógica y profunda dentro de una jerarquía como un todo. Las comparaciones entre los pares se hacen primero planteando la pregunta "¿Cuál de los dos es más importante?". La fortaleza de la preferencia es expresada en una escala de 1 a 9, lo que permite la medida dentro del mismo orden de magnitud. Para este artículo la escala de calificación se simplificó en tres opciones, de la siguiente manera:

1 SFAC S, FASB. Consultado el 3 de mayo de 2011 en: http//www.demiss.edu/coures/accy303/Chapt2,pdf 
Tabla 1. Escala de calificación²

\begin{tabular}{|c|l|}
\hline Notas & \multicolumn{1}{|c|}{ Escala Semántica } \\
\hline 1 & Igual importancia \\
\hline 2 & Más importante \\
\hline 3 & Fuertemente más importante \\
\hline
\end{tabular}

Estas comparaciones resultan en una matriz de comparaciones donde:

$$
\begin{gathered}
A_{\mathrm{ii}}=1 ; \\
\mathrm{A}_{\mathrm{ij}}=\mathrm{K} ; \\
\mathrm{A}_{\mathrm{ji}}=1 / \mathrm{k} .
\end{gathered}
$$

Tabla 2. Matriz de Preferencias ${ }^{3}$

\begin{tabular}{|r|c|c|c|}
\hline Objetivo & Indicador A & Indicador B & Indicador C \\
\hline Indicador A & 1 & 3 & 2 \\
\hline Indicador B & $1 / 3$ & 1 & $1 / 3$ \\
\hline Indicador C & $1 / 2$ & 3 & 1 \\
\hline \multicolumn{5}{c}{} \\
Inverso
\end{tabular}

El cruce de indicadores en la matriz con notas igual a 1 , es porque estos indicadores se están comparando con ellos mismos y, por lo tanto, en ellos se cumple la primera regla que es: si el indicador que está en la fila (i) es igual al que está en la columna (j), entonces, la nota que se colocará es $1\left(\mathrm{~A}_{\mathrm{ii}}=1\right)$.

Por otro lado, al comparar el indicador A con el B, el grupo consideró que el indicador A que está en la fila, es más importante que el indicador $\mathrm{B}$, que está en la columna, por tanto, la nota colocada en esta relación fue $3\left(\mathrm{~A}_{\mathrm{ij}}=\mathrm{k}\right)$.

Cuando el indicador A deja de estar en la fila y se ubica en la columna y, por tanto, el indicador B, pasa a la fila; la nota que se colocará es $1 / 3$ que es el inverso del valor hallado en la explicación anterior. Esto significa que al cambiar la posición del indicador A, este seguirá siendo importante, así se ubique

Tomado de Saaty (2005).

Tomado de Saaty (2005). en la columna, en relación a $\mathrm{B}$, ya que el $1 / 3\left(\mathrm{~A}_{\mathrm{ji}}=\right.$ $1 / \mathrm{K})$ significa que el indicador que está en la columna (A), es más importante que el de la Fila (B), en ese sentido se sigue siendo consistentes con lo dicho en el párrafo anterior.

La metodología para aplicar la herramienta AHP consta de los siguientes pasos de acuerdo con González (2009):

- La Construcción de las Prioridades: consiste en la construcción de un conjunto homogéneo de elementos con un elemento individual que representa un propósito global.

- La identificación de parejas: consiste en comparar dos indicadores dentro de una perspectiva contra un criterio dado para establecer un peso relativo. Saaty (2005) anota que la comparación puede ser realizada en términos de importancia, probabilidad o preferencia.

- La Síntesis: en este paso se combina matemáticamente todos los valores de preferencia dado a los indicadores de tal forma que se pueda determinar las prioridades. Esto se logra a partir de los valores obtenidos en el paso anterior. Los resultados que se obtienen corresponden a los valores relativos de importancia (VRI) y a las jerarquías. Ajustando los resultados para una solución normalizada se garantiza que la suma total de los valores relativos en importancia sean iguales a 1 ó $100 \%$.

- Revisar las consistencias: para lo cual se debe calcular un porcentaje que se considera como el esperado y otro que es el real. La confrontación de ambos permite determinar la calidad de las decisiones tomadas.

- Evaluar los Resultados: una vez obtenidos los índices de desempeño esperado y los reales, las prioridades son finalmente evaluadas e interpretadas.

La herramienta del AHP contribuye a resolver problemas complejos de toma de decisiones que involucran múltiples criterios e indicadores. En este sentido, la herramienta contribuye con los procesos de gestión de intangibles al interior de organizaciones 
basadas en el conocimiento como son las empresas de software.

\subsection{Balanced Scorecard (BSC)}

El Balanced Scorecard (BSC) desarrollado por Kaplan \& Norton (1997) es una herramienta que sirve para gestionar la estrategia a largo plazo y aporta una visión integral de los sistemas de medición económica en una organización, puesto que involucra aspectos financieros y no financieros.

Según Kaplan \& Norton (1997, 20), "Lo ideal hubiera sido que el modelo de contabilidad financiera se hubiera expandido hasta incorporar la valoración de los intangibles de una empresa, como son los productos y servicios de alta calidad; los empleados, expertos $y$ motivados; los procesos internos predecibles y sensibles; y unos clientes retenidos y satisfechos", desafortunadamente, la contabilidad tradicional se ha quedado rezagada frente a el nuevo escenario de los negocios en la era de información.

Por otro lado, si los intangibles y las capacidades de la empresa pudieran ser valorados dentro del modelo de la contabilidad financiera, las organizaciones fácilmente podrían divulgar a los diferentes usuarios de dicha información. También se comunican los cambios que se están dando en los intangibles y dar a conocer como esos cambios están contribuyendo con la creación de valor, pero esto no sucede.

El BSC se utiliza por empresas innovadoras como un sistema de gestión estratégica para llevar a cabo los siguientes procesos de gestión:

- Aclarar y traducir o transformar la visión y la estrategia.

- Comunicar y vincular los objetivos e indicadores estratégicos.

- Planificar, establecer objetivos y alinear las iniciativas estratégicas.

- Aumentar el feedback y la formación estratégica.

El BSC parte de la premisa que los gerentes deben gerenciar y evaluar sus negocios desde un mínimo de cuatro perspectivas: Financiera, Clientes, Proce- sos internos y Aprendizaje y Crecimiento. Las cuatro perspectivas llevan a los gerentes a desarrollar e integrar la estrategia entorno a cuatro preguntas (Kaplan \& Norton, 1997):

a) ¿Cómo nos ven los accionistas? La perspectiva financiera es medida por indicadores como flujo de caja, retorno sobre el patrimonio, participación del mercado, etc.;

b) ¿Cómo los clientes ven la empresa? La perspectiva de los clientes es medida en parte por indicadores de satisfacción de clientes, entrega a tiempo, retención de clientes, adquisición de clientes, rentabilidad del cliente, participación del mercado, participación en las compras del cliente, etc.;

c) ¿Qué procesos del negocio, la empresa debe mejorar? La perspectiva de los procesos internos es medida por indicadores tales como ciclos de tiempo, costo unitario, calidad, rotación de inventarios, gastos de investigación y desarrollo, tasas de utilización de mano de obra, etc.;

d) ¿Puede la empresa continuar innovando y aprendiendo? La perspectiva de aprendizaje y crecimiento es medida en parte por indicadores tales como cantidad y calidad de la gestión de los empleados, habilidades desarrolladas por los empleados, absentismo, gestión del conocimiento, ratio de información estratégica, número de directivos, etc.

Uno de los elementos básicos para el diseño del BSC es el Mapa Estratégico, el cual describe gráficamente la estrategia de una empresa, lo que facilita el consenso desde la alta gerencia hasta el personal de planta, consecuentemente, la comunicación efectiva de la estrategia al interior de la organización y la alineación de empresa con ella.

"El Mapa Estratégico muestra la conexión de los intangibles (el Capital Humano, la Tecnología de la Información y el Capital Organizacional: Clima, Cultura, Liderazgo) con los procesos internos de creación de valor. Estos procesos internos son los que habilitan a la Organización a entregar su propuesta de valor al cliente objetivo (Symnetics, 2003). 
Los intangibles se transforman en activos tangibles (productos y servicios) a través de la Propuesta de Valor para los clientes. Por tanto, los clientes serán atraídos, satisfechos y retenidos, si la Propuesta de Valor se ajusta a sus necesidades y expectativas. La respuesta de los clientes tendrá impactos financieros positivos para la organización.

A través de los Mapas Estratégicos, las cuatro perspectivas del BSC, se efectivizan a partir de un conjunto de objetivos estratégicos, que se conectan por relaciones causa-efecto, por medio de los cuales se describe la estrategia que debe seguir la organización. De esta manera, el modelo del BSC y el diseño de Mapas Estratégicos, convierten la visión y la estrategia de la organización en objetivos e indicadores que se articulan a las cuatro perspectivas identificadas: financiera, del cliente, del proceso interno y de aprendizaje y crecimiento. Además, permite que los directivos de una organización centren sus esfuerzos en aquellas perspectivas que se encuentran en dificultades, logrando así que la estrategia de la organización, evolucione de acuerdo a los cambios del entorno tecnológico, competitivo y de mercado, lo que impactará positivamente en la creación de valor para los clientes.

\section{Metodología y caso de estudio}

Para la aplicación de la metodología AHP, se diseñó una encuesta, teniendo en cuenta los pasos de dicha herramienta y se aplicó a tres empresas de software que hacen parte del Parque Tecnológico de Santiago de Cali. Las empresas que sirvieron de referencia para construir el caso, se caracterizan por ser pequeñas empresas, llevan contabilidad y se encuentran registradas en Cámara de Comercio.

La aplicación de la herramienta AHP se realizó en función de cinco tipos de intangibles de primer nivel identificados: Innovación y Tecnología; Capacidad de Gestión; Capacidad de los Colaboradores; Relaciones con los Clientes; Fuerza de Mercado (Ver Tabla 1).
En primer lugar se preguntó sobre la importancia de los diferentes intangibles al interior de cada una de las empresas con el fin de identificar el grado de prioridad o importancia que tenían cada uno de ellos para la empresa. En ese sentido, se construyeron matrices de preferencia y se utilizó la escala de calificación de tres opciones (1: Igual importancia; 2: Más importante; 3: Fuertemente más importante).

A cada tipo de intangible de primer nivel, se identificaron un conjunto de otros intangibles que los caracterizan, los cuales se denominaron intangibles de segundo nivel (Ver Tabla 2) y estos también fueron priorizados a través de aplicar el AHP. Cada uno de los intangibles de primer nivel se relacionó con cada una de las perspectivas del BSC, cabe anotar que en cada perspectiva fueron organizados de acuerdo a las jerarquías determinadas por el AHP. A continuación, se presenta la forma como se relacionaron los intangibles de primer nivel y las Perspectivas del BSC:

- Perspectiva Financiera: Incremento en las ventas.

- Perspectiva del Cliente: Relación con los clientes y alianzas y Fuerza de Mercado.

- Perspectiva de Procesos Internos: Innovación y tecnología y Capacidad de Gestión.

- Perspectiva de Aprendizaje y Crecimiento: Capacidad de los colaboradores.

Para cada uno de los intangibles de primer nivel, se identificaron los intangibles de segundo nivel, de acuerdo con su orden de priorización y para cada uno de estos se definió un objetivo estratégico, a partir de los cuales se construyeron los mapas de valor o estratégicos.

A partir de los resultados del BSC se construyó un Tablero de Mando para la Empresa A en el cual a cada intangible se le identifica un objetivo estratégico y se le asigna un indicador, que permite hacer un monitoreo del comportamiento de los mismos al interior de la empresa (Ver tabla 3). Los criterios para la interpretación de los indicadores son los siguientes: 
Tabla 3. Criterios de indicadores ${ }^{4}$

\begin{tabular}{|c|c|}
\hline $\begin{array}{c}\text { Criterios de interpretación } \\
\text { para los indicadores }\end{array}$ & Color \\
\hline Bueno & Verde \\
\hline Regular & Amarillo \\
\hline Pésimo & Rojo \\
\hline
\end{tabular}

Para explicar la aplicación de la herramienta Analytic Hierarchy Process (AHP) y el Balanced Scorecard (BSC) en la gestión de intangibles en empresas de software, ubicadas en Cali, Colombia, se trabajará en la forma de caso de estudio para tres empresas, las cuales tienen características particulares que se describen a continuación:

Empresa A. Desarrolladora de Software: La principal actividad de esta empresa es el Desarrollo de Software. Aunque es una pequeña empresa, cuenta con personal altamente calificado, principalmente Ingenieros de Sistemas, con estudios de posgrado, lo que le ha permitido la incorporación permanente de Ciencia, Tecnología e Innovación. Actualmente, esta empresa se encuentra desarrollando un software que contribuirá a la integración de la información procedente de diferentes áreas de una compañía (costos, inventarios, logística, personal, etc.) a través de un solo aplicativo. El desarrollo de este software ha requerido bastante tiempo e inversión en capital humano.

Empresa B. Comercializadora de Software: Esta empresa, a diferencia de la anterior, no se dedica al desarrollo de software, sino que se encarga de comercializarlo y establecer un puente entre las empresas desarrolladoras y los clientes actuales y potenciales que demandan este tipo de productos. También es una pequeña empresa y el personal es multidisciplinario, entre los que se encuentran además de ingenieros, administradores de empresas, contadores públicos y ejecutivos de ventas. Gracias a las buenas relaciones comerciales que tiene la empresa con sus clientes, al conocimiento del mercado y a las alianzas realizadas con las empresas de software que se ubican en el Parque Tecnológico de Santiago de Cali, los productos de software que comercializan han logrado insertarse de manera eficiente y oportuna en el mercado nacional e, incluso, algunos han sido comercializados en el exterior.

Empresa C. Mantenimiento y soporte de software: Esta empresa se dedica al soporte y mantenimiento tanto de software como de hardware. Sus empleados son principalmente Ingenieros electrónicos y de sistemas además cuentan con técnicos en sistemas y con un equipo de ejecutivos de ventas que se dedican a vender los servicios que presta la compañía. Gracias al excelente servicio al cliente, esta empresa ha logrado alcanzar una alta reputación y prestigio en el mercado, constituyéndose en una compañía reconocida y con un gran número de clientes.

\section{Principales resultados}

\subsection{Aplicación de la herramienta AHP}

En primer lugar se preguntó sobre la importancia de los diferentes intangibles al interior de cada una de las empresas, a través de la construcción de matrices de preferencia y utilizando la escala de calificación descrita en el marco conceptual de este artículo. Los resultados de esta calificación se presentan en la Tabla 4.

Tabla 4. Evaluación de los intangibles de Primer $\mathrm{Nive}^{5}$

\begin{tabular}{|l|c|c|c|c|c|}
\hline & $\begin{array}{c}\text { Innovación y } \\
\text { tecnología }\end{array}$ & $\begin{array}{c}\text { Capacidad de } \\
\text { gestión }\end{array}$ & $\begin{array}{c}\text { Capacidad } \\
\text { de los } \\
\text { colaboradores }\end{array}$ & $\begin{array}{c}\text { Relación con } \\
\text { los clientes y } \\
\text { alianzas }\end{array}$ & $\begin{array}{c}\text { Fuerza de } \\
\text { Mercado }\end{array}$ \\
\hline EMPRESA A & $0,403(1)$ & $0,114(2)$ & $0,194(4)$ & $0,085(5)$ & $0,203(3)$ \\
\hline EMPRESA B & $0,074(5)$ & $0,148(4)$ & $0,167(3)$ & $0,348(1)$ & $0,263(2)$ \\
\hline EMPRESA C & $0,118(5)$ & $0,121(4)$ & $0,127(3)$ & $0,261(2)$ & $0,373(1)$ \\
\hline
\end{tabular}

4 Datos de la investigación.

Fuente: Elaboración propia. 
A partir de los resultados que arrojaron las matrices de preferencia se observa en la tabla 1 que la Empresa A desarrolladora de Software, el intangible que tiene una mayor importancia o prioridad para esta empresa es el de Innovación y Tecnología; una vez que su core-bussines está representado en la capacidad de innovar permanentemente. En segundo lugar de importancia se encuentra la capacidad de gestión, siendo este un elemento importante para que su producto se desarrolle con los más altos estándares de calidad e incorpore tecnología de punta. En tercer lugar, se encuentra la Fuerza de Mercado, esta variable le permitirá posicionarse en el mercado y constituirse en una marca reconocida por su calidad e innovación. Por último, se encuentra la capacidad de los empleados y las relaciones con los clientes y alianzas, que aunque son intangibles muy importantes, para la gerencia existen otros indicadores de mayor relevancia, tal como se constata en los resultados de la priorización.

En la Empresa B, dedicada a la comercialización y distribución de productos de software, el intangible que tiene más importancia para ellas es la relación con los clientes y alianzas, pues a partir de esto pueden consolidarse y aumentar su participación en el mercado. En segundo lugar, se encuentra la Fuerza de Mercado, gracias a este intangible, la empresa logra un reconocimiento en el sector, lo que permite mantener o ganar nuevos clientes de manera sistemática. En tercer lugar, se encuentra la capacidad de los colaboradores, una vez que las personas encargadas de realizar las ventas sean eficaces, esto permitirá que el volumen de ventas se incremente permanentemente. Debido a que esta empresa no se dedica al desarrollo de software como tal, la preferencia que se tiene sobre los intangibles: Innovación y Tecnología y Capacidad de Gestión, es menor.

Por su parte la Empresa $\mathrm{C}$, al ofrecer servicios de mantenimiento y soporte técnico, la mayor preferencia para esta empresa se enfoca en la Fuerza de Mercado que puede alcanzar, pues en esta medida pueden aumentar, la base de clientes y ofrecer mayores y mejores servicios en el mercado. Por otro lado, la relación con los clientes y alianzas se constituye como el intangible en segundo lugar de preferencia. Por otro lado, los intangibles como la valoración de la capacidad de los colaboradores, capacidad de gestión y la innovación y tecnología presentan una menor preferencia.

Se evidencia que el nivel de preferencia mostrado por las empresas sobre los mismos intangibles varía de una empresa para otra, como también que existe una relación lógica entre la preferencia del intangible y la actividad principal que desarrolla el negocio. Con el fin de identificar de manera más detallada preferencia de los intangibles al interior de las empresas de software, a cada tipo de intangible, se identificaron un conjunto de intangibles de segundo nivel. En la Tabla 5 se resume los resultados del análisis realizado en cada empresa para los intangibles de segundo nivel.

En segundo lugar, se encuentra la Investigación y Desarrollo por parte de los colaboradores, pues para la Empresa A, el capital intelectual es un intangible de mucho valor, que le permite a la empresa desarrollar software que incorpore innovación y conocimiento. En tercer lugar, se encuentra la marca, pues este intangible se logra construir a través del tiempo y permite el posicionamiento y reconocimiento de la empresa en el mercado. Posteriormente se encuentra los contratos con los clientes, ya que estos son los que constituyen la creación de valor para la compañía. En quinto lugar, se ubica la capacidad de I\&D, que se relaciona de manera directa con la valoración del Intangible de primer nivel Innovación y Tecnología.

Con respecto a la Empresa $\mathrm{B}$, comercializadora y distribuidora de software, el intangible de segundo nivel, al cual le da mayor importancia es al entrenamiento y capacidad de los colaboradores, una vez que al contar con un buen equipo de ventas, tendrá una alta probabilidad de que la empresa se posicione en el mercado y muchas otras empresas la contraten para distribuir y comercializar sus productos. 
Tabla 5. Importancia de los Intangibles de Segundo Nivel ${ }^{6}$

\begin{tabular}{|c|c|c|c|}
\hline DIMENSIONES & EMPRESA A & EMPRESA B & EMPRESA C \\
\hline \multicolumn{4}{|l|}{ INNOVACIÓN Y TECNOLOGÍA } \\
\hline Tecnologías clave & $0,266(8)$ & $0,146(14)$ & $0,233(9)$ \\
\hline Capacidad de I\&D & $0,351(5)$ & $0,140(15)$ & $0,197(11)$ \\
\hline Procesos de fabricación & $0,108(19)$ & $0,077(20)$ & $0,120(18)$ \\
\hline Procesos de servicios & $0,086(21)$ & $0,373(4)$ & $0,356(4)$ \\
\hline Registro de marca & $0,189(11)$ & $0,264(7)$ & $0,095(19)$ \\
\hline \multicolumn{4}{|l|}{ CAPACIDAD DE GESTIÓN } \\
\hline Capacidad de Gestión de Activos & $0,168(14)$ & $0,469(2)$ & $0,172(12)$ \\
\hline Capacidad de control Interno & $0,117(17)$ & $0,279(6)$ & $0,128(16)$ \\
\hline Calidad en la Operación & $0,231(10)$ & $0,148(13)$ & $0,350(5)$ \\
\hline Capacidad de actualización de tecnología & $0,484(1)$ & $0,104(18)$ & $0,350(5)$ \\
\hline \multicolumn{4}{|l|}{ CAPACIDAD DE LOS COLABORADORES } \\
\hline Investigación y Desarrollo por parte de los colaboradores & $0,429(2)$ & $0,098(19)$ & $0,129(15)$ \\
\hline Capacidad de innovación de los colaboradores & $0,303(7)$ & $0,210(10)$ & $0,200(10)$ \\
\hline Conocimiento de los Colaboradores & $0,170(13)$ & $0,210(10)$ & $0,258(7)$ \\
\hline Entrenamiento y capacitación de los colaboradores & $0,098(20)$ & $0,481(1)$ & $0,413(2)$ \\
\hline \multicolumn{4}{|l|}{ RELACIÓN CON LOS CLIENTES Y ALIANZAS } \\
\hline Contratos con los clientes & $0,359(4)$ & $0,344(5)$ & $0,396(3)$ \\
\hline Participación en las compras de los clientes & $0,127(16)$ & $0,135(16)$ & $0,243(8)$ \\
\hline Derechos de distribución & $0,085(22)$ & $0,205(11)$ & $0,146(13)$ \\
\hline Contratos de Cooperación & $0,174(12)$ & $0,176(12)$ & $0,130(14)$ \\
\hline Acuerdos con los socios & $0,256(9)$ & $0,140(15)$ & $0,086(20)$ \\
\hline \multicolumn{4}{|l|}{ FUERZA DE MERCADO } \\
\hline Reputación de la compañía & $0,327(6)$ & $0,238(8)$ & $0,524(1)$ \\
\hline Lealtad de los clientes & $0,147(15)$ & $0,442(3)$ & $0,267(6)$ \\
\hline Cultura empresarial & $0,110(18)$ & $0,107(17)$ & $0,082(21)$ \\
\hline Marca & $0,417(3)$ & $0,213(9)$ & $0,427(17)$ \\
\hline
\end{tabular}

En segundo lugar, se encuentra la capacidad de gestión de los activos, ya que un manejo eficiente de los mismos, le permitirá a la empresa tener unos márgenes de ganancias más altos. En tercer lugar, se encuentra la lealtad de los clientes. Le siguen en orden de preferencia, los procesos en el servicio y los contratos con los clientes, pues dependiendo de su relación con los mismos, logrará un posicionamiento importante en el mercado.

En relación con la Empresa $\mathrm{C}$ que se dedica al soporte y mantenimiento del software, el indicador al

6 Resultados de la investigación. 
cual le otorgan mayor importancia es la Fuerza de Mercado, ya que esta le permite ganar reputación y así ampliar y afianzar las relaciones con los clientes. En segundo lugar, se encuentra el entrenamiento y capacidad de los colaboradores, en la medida que estos sean eficientes y realicen una buena labor, seguirán siendo considerados por sus clientes para servicios posteriores de soporte y mantenimiento. En tercer lugar y, al igual que las anteriores empresas, se encuentran los contratos con los clientes gracias a estos se puede lograr el crecimiento de la compañía. En cuarto lugar, se encuentra los procesos de servicios, pues esta empresa se dedica principalmente a la prestación de servicios de soporte y mantenimiento. En quinto lugar, se encuentra la capacidad de operación de calidad, ya que si todo el proceso de servicios se realiza con buenos estándares de calidad, se garantiza el posicionamiento de la compañía en el mercado.

De esta manera, se concluye que aunque las empresas presentan diferentes niveles de preferencia sobre los intangibles al interior de la organización, se observa que en algunos intangibles de segundo nivel hubo coincidencias, tal es el caso de los contratos con los clientes, el entrenamiento y capacitación de los colaboradores y los procesos de servicios.

\subsection{Aplicación del BSC}

Para cada uno de los intangibles de primer nivel, se seleccionaron dos intangibles de segundo nivel, de acuerdo con su orden de priorización, y para cada uno de estos se identificó un objetivo estratégico, con estos objetivos se construyó los mapas de valor para las tres empresas, tal como se muestra en el Anexo 1. Es de anotar que a las tres empresas les fue elaborado su respectivo mapa de valor, tan solo a la empresa $\mathrm{A}$, le fue construido el tablero de mando, esto para que los lectores tengan una idea de cómo sería el proceso hasta la elaboración del Tablero de Mando. A partir del Tablero de Mando, el tomador de decisión puede gerenciar los intangibles en razón al comportamiento de los indicadores designados para cada objetivo estratégico que se vincula con cada intangible. Los mapas de valor de las empresas B y C se encuentran en el Anexo 1.
Como se puede observar en la Tabla 3, los intangibles fueron traducidos en objetivos estratégicos, los cuáles a su vez fueron medidos por sus respectivos indicadores. Con base en el sistema de alertas creado, se observa que algunos intangibles tuvieron un comportamiento bueno, regular o pésimo. De tal forma que con los resultados de las mediciones realizadas que se vinculan con el logro o no del objetivo estratégico, los tomadores de decisión deberán establecer iniciativas estratégicas para mejorar aquellos indicadores que presenten alertas de color rojo o amarillo.

\section{Consideraciones finales}

El abordaje propuesto a través de este artículo se convierte en una primera aproximación para demostrar como a partir de la combinación de dos herramientas, una proveniente de la matemáticas (AHP) y otra de la Gestión (BSC), se puede generar un proceso que lleva a monitorear el desempeño de los intangibles al interior de una organización basada en el conocimiento, como es el caso de las empresas de software.

Los resultados de este caso de estudio dan luces para futuros trabajos en el sentido de que no solo es suficiente con la herramienta de gestión sino que también es importante poder contar con otras herramientas que complementen al gerente en cuanto a una información más integral sobre sus intangibles. Tal como lo hace el AHP que permite realizar la priorización, en grado de importancia, de los diversos activos intangibles que existen en una organización en los días de hoy. Lo anterior es importante ya que con la priorización, los gerentes podrán identificar qué intangibles son estratégicos y sobre los cuales deben estar atentos a cambios en su comportamiento, una vez que de ellos depende la creación de valor y la ventaja competitiva.

Finalmente, la integración de la herramienta AHP y el BSC resulta útil para la traducción de los intangibles en objetivos estratégicos e indicadores, los cuales permitirán la creación de valor, la implementación de la propuesta de valor, la calidad de los procesos y un personal altamente calificado. 
Tabla 3. Empresa A. Desarrolladora de Software -Tablero de Mando ${ }^{7}$

\begin{tabular}{|c|c|c|c|c|c|}
\hline \multirow{2}{*}{$\begin{array}{c}\text { PERSPECTIVA } \\
\text { FINANCIERA }\end{array}$} & \multirow[t]{2}{*}{ INTANGIBLE } & \multirow{2}{*}{$\begin{array}{l}\text { OBJETIVOS ESTRATÉGICOS } \\
\text { INCREMENTO DE LAS VENTAS }\end{array}$} & \multicolumn{2}{|l|}{ INDICADORES } & \multirow[b]{2}{*}{ COLOR } \\
\hline & & & $\begin{array}{c}\text { \% AUMENTO EN LAS } \\
\text { VENTAS }\end{array}$ & $5 \%$ & \\
\hline \multirow{4}{*}{$\begin{array}{c}\text { PERSPECTIVA } \\
\text { DEL CLIENTE (Inter- } \\
\text { nos y } \\
\text { Externos) }\end{array}$} & Marca & Reconocer la Marca de la Empresa & $\begin{array}{l}\% \text { de encuestas contestadas favo- } \\
\text { rablemente }\end{array}$ & $40 \%$ & Amarillo \\
\hline & Contratos con los clientes & Satisfacer las necesidades de los clientes & $\begin{array}{l}\% \text { de encuestas contestadas favo- } \\
\text { rablemente }\end{array}$ & $45 \%$ & Amarillo \\
\hline & Reputación de la compañía & Elaborar productos con calidad & $\begin{array}{l}\% \text { de reclamos y quejas sobre los } \\
\text { productos }\end{array}$ & $30 \%$ & Amarillo \\
\hline & Acuerdos con los socios & Satisfacer las necesidades de los socios & $\begin{array}{l}\text { No. de acuerdos establecidos con } \\
\text { los socios }\end{array}$ & $40 \%$ & Rojo \\
\hline \multirow{4}{*}{$\begin{array}{l}\text { PERSPECTIVA } \\
\text { DE PROCESOS } \\
\text { INTERNOS }\end{array}$} & $\begin{array}{l}\text { Capacidad actualización } \\
\text { de tecnología }\end{array}$ & $\begin{array}{l}\text { Mejorar procesos de capacitación en actuali- } \\
\text { zación de tecnología }\end{array}$ & No. de procesos mejorados & 4 & Verde \\
\hline & Capacidad de I\&D & $\begin{array}{l}\text { Establecer procesos que mejoren la capacidad } \\
\text { en I \&D }\end{array}$ & $\begin{array}{l}\% \text { de incremento en productos in- } \\
\text { novadores }\end{array}$ & $60 \%$ & Verde \\
\hline & Tecnologías clave & $\begin{array}{l}\text { Mejorar los procesos relacionados con el uso } \\
\text { de tecnologías clave }\end{array}$ & No. de procesos mejorados & 3 & Amarillo \\
\hline & Calidad en la operación & Mejorar los procesos de operación de la calidad & Número de procesos certificados & 4 & Amarillo \\
\hline \multirow{2}{*}{$\begin{array}{l}\text { PERSPECTIVA } \\
\text { DE APRENDIZAJE Y } \\
\text { CRECIMIENTO }\end{array}$} & $\begin{array}{l}\text { Investigación y desarrollo } \\
\text { por parte de los colabo- } \\
\text { radores }\end{array}$ & $\begin{array}{l}\text { Realizar cursos de capacitación que aumen- } \\
\text { ten las habilidades en I+D por parte de los } \\
\text { colaboradores }\end{array}$ & \% de empleados capacitados & $90 \%$ & Verde \\
\hline & $\begin{array}{l}\text { Capacidad de innovación } \\
\text { por parte de los colabo- } \\
\text { radores }\end{array}$ & $\begin{array}{l}\text { Crear un ambiente organizacional que invite } \\
\text { a la innovación }\end{array}$ & $\%$ de empleados bonificados & $40 \%$ & Amarillo \\
\hline
\end{tabular}

\section{Referencias}

Arthur Andersen \& Co. (1992) The valuation of Intangible Assets, Special Report. London, The Economist Intelligence Unit.

Belkaoui, A. (1992). Accounting Theory. London Academic Press.

Cañibano et. Al (1999). La Relevancia de los intangibles para la Valoración y la Gestión de Empresas: Revisión de la Literatura en: Revista Española de Financiación y Contabilidad, 100: 17-88.

Canibaño, L. et. al. (2000). Accounting for Intangibles: A literature Review en: Journal of Accounting Literature: 102- 130.

Chiesa, V. et. al. (2005). The valuation of technology in buy cooperate-sell decisions en: European Journal of Innovation Management: 5-30.

Egginton, D. (1990). Towards some principles for intangible asset accounting en: Accounting and Business Research, 22, spring: 85-102.

González, P. (2009). La integración del Balanced Scorecard (BSC) y el Analytic Hierarchy Process (AHP) para efectos de jerarquizar medidas de desempeño y toma de decisión en una institución financiera. En: Revista Universo Contábil, 5, (2): 87-105.

Hendriksen, E. \& Van Breda, M.F. (1992). Accounting Theory. 5th ed, Burr Ridge, Irwin.

Hendriksen, E. (1982). Accounting Theory. 4th ed, Burr Ridge, Irwin.
Kaplan, R. \& Norton, D. (1997) Cuadro de Mando Integral (The Balanced ScoreCard). Harvard Business School Press. Barcelona, Gestión 2000.

King, K. (2003). The value of intellectual property, intangible assets an goodwill. EMI: 1-3. www.valuation-consulting.co.uk.

Lev, B. (2003). Remarks on the measurement, Valuation and Reporting of Intangible Assets en: Economic Policy Review:17-22.

Lev, B. (2004). Sacar más ventaja de los intangibles en: Harvard Business Review América Latina.

Marr, B., Gray, D. \& Neely, A. (2003). Why do firms measure their intellectual capital? Journal of IntellectualCapital, 4 (4): 441-464.

OECD (1992 a). Technology and the Economy. The Key Relatioships, Paris.

OECD (1992 b). OECD proposed guidelines for collecting and interpreting technological innovation data Oslo Manual Paris.

Ordoñez, P. (2000). Importancia estratégica de la medición del capital intelectual en las organizaciones. Extraído el 28 de Febrero de 2009 disponible en: http://www.injef.com/revista/ empresas/pop_991217.htm

Reilly, R. (1994). Valuation of intangible assets for bankruptcy and reorganization purpose en: Ohio CPA Journal:25-30.

Saaty, T. (2005). Theory and Applications of the Analytic Network Process. RWS Publications.

7 Datos de la investigacion. 
Symnetics Colombia. (2003). Balanced Scorecard Collaborative y Symnetics. Santiago de Chile.

Stickney, C. \& Weil, R. (1994). Financial Accounting, Forth Worth, The Dryden Press.
Volsselman, W. (1998). Initial guidelintes for the collection and comparison of data on intangible investment. Netherlands Central Bureauf of Statistics \& OECD.

White, G. Sondhi, A. \& Fried, D. (1994). The analysis and uses of financial statements. New York, John Wiley and Sons.

\section{Anexo $1^{8}$}

Aplicación de la Herramienta Balanced Scorecard. Empresa A. Desarrolladora de Software

\begin{tabular}{|c|c|c|}
\hline $\begin{array}{l}\text { PERSPECTIVA } \\
\text { FINANCIERA }\end{array}$ & \multicolumn{2}{|c|}{ INCREMENTO DE LAS VENTAS } \\
\hline \multirow{4}{*}{$\begin{array}{l}\text { PERSPECTIVA } \\
\text { DEL CLIENTE } \\
\text { (Internos y } \\
\text { Externos) }\end{array}$} & Marca & Reconocer la Marca de la empresa \\
\hline & Contratos con los clientes & Satisfacer las necesidades de los clientes \\
\hline & Reputación de la compañía & Elaborar productos con calidad \\
\hline & Acuerdos con los socios & Satisfacer las necesidades de los socios \\
\hline \multirow{4}{*}{$\begin{array}{c}\text { PERSPECTIVA } \\
\text { DE } \\
\text { PROCESOS } \\
\text { INTERNOS }\end{array}$} & $\begin{array}{l}\text { Capacidad de actualización } \\
\text { de tecnología }\end{array}$ & $\begin{array}{l}\text { Mejorar los procesos de capacitación en } \\
\text { actualización de tecnoloaía }\end{array}$ \\
\hline & Capacidad de I\&D & $\begin{array}{l}\text { Establecer procesos que mejoren la capacidad } \\
\text { en I\&n }\end{array}$ \\
\hline & Tecnologías claves & $\begin{array}{l}\text { Mejorar los procesos relacionados con el uso de } \\
\text { tecnología clave }\end{array}$ \\
\hline & Calidad en la operación & Mejorar los procesos de operación de la calidad \\
\hline \multirow{2}{*}{$\begin{array}{c}\text { PERSPECTIVA } \\
\text { DE } \\
\text { APRENDIZAJE } \\
\text { Y } \\
\text { CRECIMIENTO }\end{array}$} & $\begin{array}{l}\text { Investigación y desarrollo por } \\
\text { parte de los colaboradores }\end{array}$ & $\begin{array}{l}\text { Realizar cursos de capacitación que aumenten } \\
\text { las habilidades en Investigación y Desarrollo por } \\
\text { parte de los colaboradores }\end{array}$ \\
\hline & $\begin{array}{l}\text { Capacidad de innovación por } \\
\text { parte de los colaboradores }\end{array}$ & $\begin{array}{l}\text { Crear un ambiente organizacional que invite a la } \\
\text { innovación }\end{array}$ \\
\hline
\end{tabular}

8 Fuente: Elaboración propia. 


\section{Anexo $2^{9}$}

Aplicación de la Herramienta Balanced Scorecard

Empresa B. Comercializadora de Software

\begin{tabular}{|c|c|c|}
\hline $\begin{array}{l}\text { PERSPECTIVA } \\
\text { FINANCIERA }\end{array}$ & \multicolumn{2}{|c|}{ INCREMENTO EN LAS VENTAS } \\
\hline \multirow{4}{*}{$\begin{array}{l}\text { PERSPECTIVA } \\
\text { DEL CLIENTE }\end{array}$} & Lealtad de los clientes & Mejorar el servicio al cliente \\
\hline & Contratos con los clientes & Satisfacer las necesidades de los clientes \\
\hline & Reputación de la compañia & Elaborar productos con calidad \\
\hline & Marca & Posicionar la Marca de la empresa \\
\hline \multirow{4}{*}{$\begin{array}{l}\text { PERSPECTIVA } \\
\text { DE PROCESOS } \\
\text { INTERNOS }\end{array}$} & $\begin{array}{l}\text { Capacidad de gestión de } \\
\text { activos }\end{array}$ & $\begin{array}{l}\text { Mejorar los procesos de capacitación en gestión } \\
\text { de los activos }\end{array}$ \\
\hline & Procesos de servicios & $\begin{array}{l}\text { Establecer procesos que mejoren los servicios } \\
\text { de la compañia }\end{array}$ \\
\hline & Capacidad de control interno & $\begin{array}{l}\text { Mejorar los procesos relacionados con el control } \\
\text { interno }\end{array}$ \\
\hline & Registro de marca & $\begin{array}{l}\text { Mejorar los procesos de registro de marca de los } \\
\text { productos de la compañía }\end{array}$ \\
\hline \multirow{3}{*}{$\begin{array}{c}\text { PERSPECTIVA } \\
\text { DE } \\
\text { APRENDIZAJE } \\
\text { Y } \\
\text { CRECIMIENTO }\end{array}$} & $\begin{array}{l}\text { Entrenamiento y capacitación } \\
\text { de los colaboradores }\end{array}$ & $\begin{array}{l}\text { Realizar cursos permanentes de capacitación y } \\
\text { actualización para los colaboradores de la } \\
\text { compañía }\end{array}$ \\
\hline & $\begin{array}{l}\text { Conocimiento de los } \\
\text { colaboradores }\end{array}$ & $\begin{array}{l}\text { Fomentar entre los colaboradores procesos de } \\
\text { aprendizaje y mejoramiento continuo }\end{array}$ \\
\hline & $\begin{array}{l}\text { Capacidad de innovación por } \\
\text { parte de los colaboradores }\end{array}$ & $\begin{array}{l}\text { Crear un ambiente organizacional que invite a la } \\
\text { innovación }\end{array}$ \\
\hline
\end{tabular}

9 Fuente: Elaboración propia. 


\section{Anexo $3^{10}$}

Aplicación de la Herramienta Balanced Scorecard

Empresa C. Mantenimiento y soporte de software

\begin{tabular}{|c|c|c|}
\hline $\begin{array}{l}\text { PERSPECTIVA } \\
\text { FINANCIERA }\end{array}$ & \multicolumn{2}{|c|}{ INCREMENTO EN LAS VENTAS } \\
\hline \multirow{4}{*}{$\begin{array}{l}\text { PERSPECTIVA } \\
\text { DEL CLIENTE }\end{array}$} & Reputación de la compañía & Elaborar productos con calidad \\
\hline & Contratos con los clientes & Satisfacer las necesidades de los clientes \\
\hline & Lealtad de los clientes & Mejorar el servicio al cliente \\
\hline & $\begin{array}{l}\text { Participación en las compras } \\
\text { de los clientes }\end{array}$ & Atraer nuevos clientes \\
\hline \multirow{4}{*}{$\begin{array}{l}\text { PERSPECTIVA } \\
\text { DE } \\
\text { PROCESOS } \\
\text { INTERNOS }\end{array}$} & Procesos de servicios & $\begin{array}{l}\text { Establecer procesos que mejoren los servicios } \\
\text { de la compañía }\end{array}$ \\
\hline & $\begin{array}{l}\text { Capacidad de actualización } \\
\text { de tecnología }\end{array}$ & $\begin{array}{l}\text { Mejorar los procesos de capacitación en } \\
\text { actualización de tecnología }\end{array}$ \\
\hline & Calidad en la operación & Mejorar los procesos de operación de calidad \\
\hline & Tecnologías clave & $\begin{array}{l}\text { Mejorar los procesos relacionados con el uso de } \\
\text { tecnología clave }\end{array}$ \\
\hline \multirow{2}{*}{$\begin{array}{c}\text { PERSPECTIVA } \\
\text { DE } \\
\text { APRENDIZAJE } \\
\mathbf{Y} \\
\text { CRECIMIENTO }\end{array}$} & $\begin{array}{l}\text { Entrenamiento y capacitación } \\
\text { de los colaboradores }\end{array}$ & $\begin{array}{l}\text { Realizar cursos permanentes de capacitación y } \\
\text { actualización para los colaboradores de la } \\
\text { compañía }\end{array}$ \\
\hline & $\begin{array}{l}\text { Conocimiento de los } \\
\text { colaboradores }\end{array}$ & $\begin{array}{l}\text { Fomentar entre los colaboradores procesos de } \\
\text { aprendizaje y mejoramiento continuo }\end{array}$ \\
\hline
\end{tabular}

10 Fuente: Elaboración propia. 